\title{
Clinicopathological characteristics of thrombospondin type 1 domain-containing 7A-associated membranous nephropathy
}

\author{
Shigeo Hara ${ }^{1,2}$ (1) $\cdot$ Takahiro Tsuji $^{3}$ - Yuichiro Fukasawa ${ }^{3}$ - Satoshi Hisano ${ }^{4}$ - Satoshi Morito ${ }^{5}$ Toshiki Hyodo $^{2,6}$. \\ Shunsuke Goto ${ }^{7}$. Shinichi Nishi ${ }^{7}$. Akihiro Yoshimoto ${ }^{8} \cdot$ Tomoo Itoh $^{2}$
}

Received: 3 October 2018 / Revised: 24 February 2019 / Accepted: 4 March 2019 / Published online: 14 March 2019

(C) The Author(s) 2019

\begin{abstract}
Thrombospondin type 1 domain-containing 7A (THSD7A) is a recently identified target antigen of idiopathic membranous nephropathy (iMN). The clinicopathological characteristics of THSD7A-associated MN are poorly characterised due to low prevalence among MN patients. Among 469 consecutive cases of pathologically confirmed MN diagnosed at four centres in Japan, 14 cases were confirmed positive for THSD7A by immunohistochemistry (3.0\%). The prevalence of THSD7A-associated MN tended to be higher in northern Japan. Most cases demonstrated nephrotic-range proteinuria (12/14 cases, $86 \%)$. In two patients, cancer was detected at the time of renal biopsy (small-cell carcinoma of the lung and prostatic adenocarcinoma with neuroendocrine differentiation). Both tumours were negative for THSD7A. Four patients had concurrent or previous incidence of allergic diseases, including one patient with Kimura's disease. Pathological analysis of kidney biopsy tissue revealed slight mesangial cell proliferation in three cases and spike formation in one case. Immunofluorescence studies demonstrated that IgG subclass was mainly IgG4dominant/codominant (12/13, 92\% cases), while the case with prostatic cancer had an IgG2-dominant distribution. The immunostaining profile for components of the lectin complement pathways was not significant in three cases including two patients with malignancy. One case was dual positive for THSD7A and PLA2R. Of 10 cases with known clinical follow-up data, 6 demonstrated reduced serum creatinine and 8 presented reduced proteinuria. In summary, although the major IgG phenotype was usually IgG4dominant/codominant, clinical background was otherwise heterogeneous. Further investigation of regional differences in THSD7Aassociated MN prevalence may reveal genetic and environmental risk factor and associated pathogenic mechanisms.
\end{abstract}

Keywords Allergic disorder $\cdot$ IgG subclass $\cdot$ Malignancy $\cdot$ Membranous nephropathy $\cdot$ THSD7A

\section{Introduction}

Membranous nephropathy (MN) is the major cause of adultonset idiopathic nephrotic syndrome. Pathologically, $\mathrm{MN}$ is characterised by subepithelial immune complex deposition, causing spike formation or a bubbling appearance in glomerular basement membranes under light microscopic examination. Membranous nephropathy associated with other diseases

Electronic supplementary material The online version of this article (https://doi.org/10.1007/s00428-019-02558-0) contains supplementary material, which is available to authorized users.

Shigeo Hara

shigeo_hara@kcho.jp

1 Department of Diagnostic Pathology, Kobe City Medical Center General Hospital, 2-1-1, Minatojima minamimachi, Chuo-ku, Kobe 650-0047, Japan

2 Department of Diagnostic Pathology, Kobe University Graduate School of Medicine, Kobe, Japan

3 Department of Pathology, Sapporo City General Hospital, Sapporo, Japan
4 Department of Pathology, Fukuoka University, Fukuoka, Japan

5 Department of Pathology, Rakuwakai Otowa Hospital, Yamashina, Japan

6 Department of Diagnostic Pathology, Kakogawa Medical Center, Kakogawa, Japan

7 Department of Nephrology, Kobe University Graduate School of Medicine, Kobe, Japan

8 Department of Nephrology, Kobe City Medical Center General Hospital, Kobe, Japan 
is generally termed 'secondary' MN (sMN). Autoimmune diseases, drugs, infections and malignant disease are the established causes of sMN [1], while MN that lacks specific clinical aetiology has been termed 'idiopathic' MN (iMN). Differentiation between iMN and sMN is a critical step for determining the optimal treatment strategy.

Recent studies have revealed the presence of autoantibodies that recognises specific target antigens in iMN. The M-type phospholipase A2 receptor (PLA2R) was the first identified major target protein. It is associated with 70-80\% of iMN cases in Western countries [2-4], and with $50-60 \%$ of cases in Japan [5-7]. There is considerable evidence to indicate that anti-PLA2R antibody levels can serve as a biomarker for assessing MN disease activity and treatment efficacy, as high anti-PLA2R antibody titre is associated with weaker response to immunosuppressive drugs, longer time to remission [8] and increased risk of kidney failure [9, 10]. Furthermore, high anti-PLA2R antibody levels accurately predicted recurrence of MN following renal transplant allograft [11].

Thrombospondin type-1 domain-containing 7A (THSD7A) was described as the second major antigenic target of autoantibodies in iMN [12]. Compared with PLA2Rassociated $\mathrm{MN}$, the prevalence of THSD7A-associated MN is generally low, ranging from 2.0 to $13.6 \%$ of iMN cases [12-15]. Recent studies have also shown a possible association of THSD7A-associated MN with malignancy $[14,16]$. However, the low prevalence of THSD7A-associated MN has hindered further examination of clinicopathological features. In this retrospective study, we assessed the clinical and pathological profile of THSD7A-associated MN in Japan.

\section{Materials and methods}

\section{Patients}

Renal biopsy specimens from 469 consecutive patients with pathologically diagnosed $\mathrm{MN}$, including both primary and secondary cases, were collected from four centres in Japan (79 from Kobe City Medical Center General Hospital, 106 from Kobe University Hospital, 98 from Sapporo City General Hospital and 186 from Fukuoka University Hospital). Cohorts treated at Kobe City Medical Center General Hospital and Kobe University Hospital were diagnosed from April 2009 to March 2018, while those treated at Sapporo City General Hospital and Fukuoka University Hospital were diagnosed from April 2012 to March 2016. Immunohistological examinations for THSD7A were performed on paraffin sections from each patient, and 14 were identified as THSD7A-positive (positivity rate of 3.0\%). The following clinical characteristics and laboratory data recorded at the time of renal biopsy were obtained from the patient database of each institution: age, sex, serum creatinine level, proteinuria $(\mathrm{g} / \mathrm{gCr})$ and incidence of other diseases such as malignancy, autoimmune diseases and infection. The time elapsed between clinical disease onset and pathological diagnosis (months) was also examined. Treatment and clinical follow-up data were also examined. All the data analyses were performed at the Department of Diagnostic Pathology, Kobe City Medical Center General Hospital. The institutional review board of Kobe City Medical Center General Hospital approved the study protocol (No. 180635).

\section{Histological analysis}

Standard processing protocols were performed for light, immunofluorescence and electron microscopic examination. Histological evaluation was performed on sections stained with haematoxylin and eosin, periodic acid-Schiff, the Masson trichrome and periodic acid methenamine silver stain. Some sections were stained with the Elastica-Masson trichrome instead of Masson trichrome according to the institutional staining protocol. The following glomerular histological features were evaluated by light microscopic examination: number of total and sclerosed glomeruli (\% sclerotic glomeruli), mesangial and endocapillary hypercellularity (presence or absence), glomerular crescent (presence or absence) and spikes in glomerular basement membrane (presence or absence).

\section{Immunohistological studies of THSD7A}

Immunostaining of THSD7A was performed according to a protocol reported previously [17]. Briefly, paraffin sections were cut at $3 \mu \mathrm{m}$, deparaffinised and incubated for $25 \mathrm{~min}$ at $95{ }^{\circ} \mathrm{C}$ and $\mathrm{pH} 9$ for antigen retrieval. Sections were then incubated with anti-THSD7A (1:800, Sigma-Aldrich, Tokyo, Japan) for $20 \mathrm{~min}$ at room temperature, followed by incubation in secondary antibodies. Granular THSD7A staining on glomerular capillaries was counted as positive, while faint positivity on glomerular capillaries and dot-like perinuclear staining were considered negative (supplementary fig. 1). In cases with concurrent malignancy, paraffin sections of tumour tissues were also examined by THSD7A immunostaining.

\section{Immunofluorescence studies}

Immunofluorescence studies were performed on frozen sections with antibodies against $\mathrm{IgG}, \operatorname{IgA}, \operatorname{IgM}, \mathrm{C} 1 \mathrm{q}$ and $\mathrm{C} 3$. PLA2R immunofluorescence studies were also performed using frozen sections according to the procedures in a previous report [7]. For cases that did not contain glomeruli in frozen sections, PLA2R was immunostained using paraffin sections [18]. Intense granular PLA2R positivity comparable to positive controls was considered immunopositive. IgG subclass analysis of glomerular deposits was performed by 
treating frozen sections with antibodies against $\operatorname{IgG} 1, \operatorname{IgG} 2$, IgG3 and IgG4 (each 1:100, Invitrogen, Camarillo, CA, USA), followed by fluorescein isothiocyanate (FITC)-conjugated secondary antibodies (1:200, Invitrogen). Immunostaining for components of the lectin complement pathway was performed on frozen sections using the following antibodies: anti-ficolin 1, anti-ficolin 3, anti-mannosebinding lectin-associated serine protease (MASP) 1/3 and anti-MASP2 (1:50, Hycult Biotech., Wayne, PA, USA). For semiquantitative assessment, immunofluorescence results were scored according to a five-grade scale from 0 to $3+(0$, negative; $<1+$, sparse; $1+$, weak; $2+$, moderate; $3+$, strong).

\section{Electron microscopic studies}

In cases assessed by electron microscopy, ultrastructural findings of the MN stage were scored based on the Ehrenreich and Churg classification [19].

\section{Results}

Of 469 consecutive cases of pathologically confirmed MN, 14 were immunopositive for THSD7A (3.0\%). Among these 14 cases, 4 each were diagnosed at Kobe University Hospital and Kobe City Medical Center General Hospital and the remaining 6 at Sapporo City General Hospital. No THSD7Aassociated MN cases were diagnosed at Fukuoka University Hospital. Therefore, THSD7A-positivity rate differed among the four institutions as follows: Sapporo City General Hospital, 10.2\%; Kobe University, 3.8\%; Kobe City Medical Center General Hospital, 5.0\%; Fukuoka University, 0\% (supplementary fig. 2). Table 1 summarises the clinical characteristics of all 14 THSD7A-associated MN cases. Average age was 64.0 (range, 42 to 79 ) years and male to female ratio was 9:5. Average time elapsed between clinical disease onset and pathological diagnosis was 3.4 (range, 2 to 8 ) months. Average serum creatinine and proteinuria levels were 0.84 (range, 0.53 to 1.40 ) $\mathrm{mg} / \mathrm{dl}, 7.59$ (range, 0.37 to 16.1 ) $\mathrm{g} / \mathrm{gCr}$ and 4.0 (range, 0.37 to 11.6 ) g/day, respectively. Allergic disorders were concurrently or previously diagnosed in four cases (cases 9-12 in Table 1). Case 9 had allergic conjunctivitis and Kimura's disease that involved subcutaneous tissue of the supraclavicular region. Case 10 had a remote history (7 years prior to renal biopsy) of eosinophilic pneumonitis and asthma. The other two cases were diagnosed with asthma 2 years (case 11) and 1 year (case 12) before renal biopsy. In addition, two cases were accompanied by malignancy at the time of renal biopsy, one (case 13) with lung small-cell carcinoma and the other (case 14) with prostatic adenocarcinoma with neuroendocrine differentiation.

Table 2 summarises the findings from glomerular histopathology. The mean proportion of sclerotic glomeruli (\% of total) was $12.1 \%$ (range, 0 to $36.8 \%$ ). Three cases showed slight mesangial cell proliferation. There were no cases with endocapillary hypercellularity or crescent formation. Spike formation on the glomerular basement membrane was observed in 1 case. Electron microscopic samples were available for 9 cases, 7 of which were classified as Ehrenreich and Churg stage I and 2 as stage I-II. Tumour tissues from cases with malignancy (case 13 and 14) were negative for THSD7A (not shown). Likewise, in case 9 with comorbid Kimura's disease, neck subcutaneous tissue was negative for THSD7A (not shown).

The heatmap plot in Fig. 1 illustrates the immunofluorescence study results. All the cases were positive for $\mathrm{IgG}$, with moderate to strong positivity in 11 cases. Twelve cases were C3-positive, of which 3 cases exhibited moderate intensity staining. Six cases were IgA-positive, of which four showed moderate immunofluorescence intensity and five cases were IgM-positive. Two cases were weakly positive for C1q. One patient was PLA2R-positive (case 2, dual positive for both PLA2R and THSD7A) (Fig. 2). Among 13 cases in which IgG subclass was examined, 12 cases showed an IgG4-dominant/codominant phenotype. Alternatively, the one case with prostatic cancer had an IgG2-dominant IgG subclass profile. Figure 3 presents representative images of immunostaining for IgG subclass.

To further characterise the pathophysiological background of THSD7A-associated MN, frozen sections were immunostained for components of the lectin complement pathway where additional frozen sections were available; cases 2, 3 and 10 were excluded from this study because of no residual samples for frozen sections. In cases 1, 4-8, 11 and 12, all components of the lectin complement pathway were positive (supplementary fig. 3); MASP 2 demonstrated weaker immunostaining than other components. In case 9 who had Kimura's disease, MASP1/3 was negative. Ficolin 1 and MASP 2 was weakly positive. In cases 13 and 14, where malignancy was detected at the time of biopsy, IF staining for the components of lectin complement pathway was less significant, with slight staining of ficolin 1 in case 13 and ficolin 3 in case 14 .

Clinical follow-up data were available for 10 patients (Table 3). These patients were monitored for 40.0 months on average (range, 3.1 to 62.6 months). All the patients with known treatment received prednisone therapy, and 5 patients also received an angiotensin II receptor blocker. Atorvastatin was administrated to two patients and one patient was treated with a calcineurin inhibitor. Six patients demonstrated reduced serum creatinine and eight patients demonstrated reduced proteinuria at the last follow-up, while one patient (case 1) still showed nephroticrange proteinuria. Case 14 did not receive treatment for MN because of advanced prostatic cancer and, thus, showed persistent nephrotic-range proteinuria. 
Table 1 Summary of clinical characteristics

\begin{tabular}{|c|c|c|c|c|c|c|c|}
\hline Case & Age & Sex & $\begin{array}{l}\text { Time from disease } \\
\text { onset to diagnosis (m) }\end{array}$ & $\mathrm{Cr}(\mathrm{mg} / \mathrm{dl})$ & Proteinuria $(\mathrm{g} / \mathrm{gCr})$ & Proteinuria (g/day) & Other diseases \\
\hline 1 & 71 & $\mathrm{~F}$ & 2 & 0.53 & 16.1 & 2.75 & - \\
\hline 2 & 68 & M & 3 & 1.16 & 7.37 & 11.6 & - \\
\hline 3 & 73 & M & 4 & 1.4 & 12 & 4.44 & - \\
\hline 4 & 68 & $\mathrm{~F}$ & NA & 1.14 & 6.68 & 6.41 & - \\
\hline 5 & 64 & M & NA & 1.02 & 5.21 & NA & - \\
\hline 6 & 61 & M & NA & 0.91 & 7.06 & 2.64 & - \\
\hline 7 & 79 & $\mathrm{~F}$ & NA & 0.55 & 9.96 & 4.43 & - \\
\hline 8 & 51 & M & 3 & 0.87 & 8.6 & NA & - \\
\hline 9 & 42 & $\mathrm{~F}$ & 2 & 0.53 & 0.37 & 0.37 & Allergic conjunctivitisKimura's disease \\
\hline 10 & 59 & M & 5 & 0.66 & 5.12 & 1.4 & eosinophilic pneumonitis asthma (52) \\
\hline 11 & 62 & $\mathrm{~F}$ & NA & 0.54 & 15.23 & NA & asthma (60) ${ }^{\#}$ \\
\hline 12 & 65 & M & NA & 0.91 & 1.68 & 1.29 & asthma (64) $\#$ \\
\hline 13 & 73 & M & 2 & 0.94 & 6.69 & 2.8 & Lung small-cell carcinoma \\
\hline 14 & 60 & M & 8 & 0.64 & 4.23 & 5.38 & $\begin{array}{l}\text { Prostatic adenocarcinoma with } \\
\text { neuroendocrine differentiation }\end{array}$ \\
\hline Average & 64.0 & $M: F=9: 5$ & 3.4 & 0.84 & 7.59 & 4.0 & \\
\hline
\end{tabular}

$\mathrm{Cr}$ creatinine, $\mathrm{F}$ female, $M$ male, $m$ months, $N A$ not assessed

\# Age at diagnosis (years)

\section{Discussion}

The prevalence of THSD7A-associated $\mathrm{MN}$ in our entire Japanese cohort was 3.0\%, comparable with previous reports $[14,15]$. Thus, THSD7A-associated MN appears to be a relatively rare phenotype in general. However, we found regional differences in the prevalence of THSD7A-associated MN, with a higher rate in the cohort from Sapporo City General Hospital. Sapporo City General Hospital receives renal biopsy specimens from a northern region of Japan (Hokkaido prefecture). Alternatively, no cases were detected from Fukuoka University Hospital, which receives renal biopsy specimens from a southwestern region of Japan (Kyushu Island). These regional differences suggest the presence of subpopulations with higher and lower susceptibility for THSD7A-associated MN. Similarly, regional differences in PLA2R-associated MN prevalence have been documented in Japan and other countries [5-7], possibly reflecting (as yet unknown) genetic,
Table 2 Summary of glomerular pathological findings

\begin{tabular}{lllllll}
\hline Case & $\begin{array}{l}\text { Glomerular } \\
\text { sclerosis }(\%)\end{array}$ & $\begin{array}{l}\text { Endocapillary } \\
\text { hypercellularity }\end{array}$ & $\begin{array}{l}\text { Mesangial cell } \\
\text { proliferation }\end{array}$ & Crescent & Spike & EM \\
\hline 1 & 15.2 & - & - & - & - & I \\
2 & 13.3 & - & - & - & - & NA \\
3 & 28.2 & - & - & - & - & NA \\
4 & 0 & - & - & - & - & NA \\
5 & 10 & - & - & - & - & I \\
6 & 8.3 & - & - & - & + & NA \\
7 & 7.7 & - & - & - & - & I \\
8 & 7.1 & - & + & - & - & NA \\
9 & 0 & - & + & - & - & I \\
10 & 10 & - & - & - & - & I-II \\
11 & 20 & - & - & - & - & I-II \\
12 & 36.8 & - & - & - & - & I \\
13 & 0 & - & - & - & - & I \\
14 & 13.3 & - & - & & - & - \\
\hline
\end{tabular}

$E M$ electron microscopy, $N A$ not assessed 
Fig. 1 Heat map summary of immunoglobulin, complement and IgG subclass immunostaining profiles. Immunostaining was graded according to a semiquantitative five-grade scale ranging from 0 to $3+$. Most cases were positive for $\mathrm{IgG}$ and $\mathrm{C} 3$. All but one case was IgG4-dominant/ codominant. Case 2 was dual positive for PLA2R and THSD7A. NA, not assessed

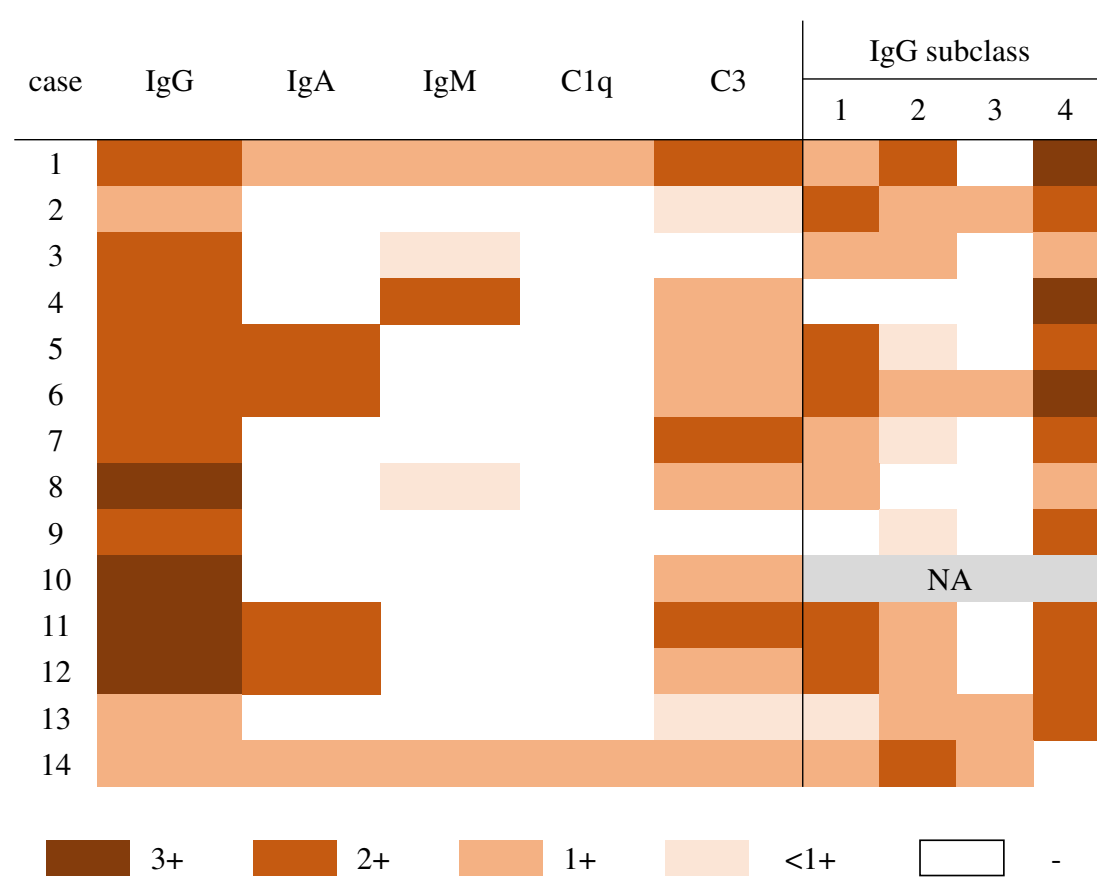

environmental and (or) lifestyle factors [7, 20, 21]. Unfortunately, the limited number of THSD7A-associated $\mathrm{MN}$ cases within a given study population hinders mechanistic investigations into these regional difference in prevalence. Moreover, our study also demonstrated substantial variation in disease features, including comorbidity and histopathological manifestations. Thus, multicentre and possibly transnational databases should be established to fully characterise the demographic and clinical features of THSD7A-associated MN.

In this study, we did not set exclusion criteria for enrolment, such as restriction to iMN cases, due to the expected rarity of THSD7A-associated MN. Therefore, the total prevalence of THSD7A-associated MN reflects the combined rate in both $\mathrm{iMN}$ and $\mathrm{SMN}$, in contrast with previous studies that focused on iMN $[12,13,15]$. To reflect recent findings on the pathogenesis of MN, the terms 'PLA2R-positive/negative $\mathrm{MN}$ ' and 'THSD7A-positive/negative MN' have been proposed [22] to replace iMN and sMN. Similar to the recently established aetiology/pathogenesis-based systems for classification and diagnosis of glomerulonephritis [23], further elucidation of the pathogenesis and clinical management of PLA2R- and THSD7A-associated MN may lead to revised nomenclature for $\mathrm{MN}$.

The present study also presents the first documented case of THSD7A-associated MN with comorbid Kimura's disease (case 9), a chronic inflammatory disorder mainly involving subcutaneous tissues of the head and neck region. The pathophysiology of Kimura's disease remains elusive, although an aberrant immune reaction to a yet unknown antigen has been proposed. Although this is the first known case of comorbidity with confirmed THSD7A-associated MN, several reports have described a possible association of Kimura's diseases with MN [24-28]. Most such cases were reported from Asian countries, including Japan and China, reflecting the endemic regions of Kimura's disease. Okura et al. reported a case of Kimura's disease with MN characterised by glomerular IgG4 and PLA2R positivity, providing the first evidence of PLA2R-associated MN with comorbid Kimura's disease [29].
Fig. 2 Membranous nephropathy with dual positivity for both PLA2R and THSD7A (case 2). a PLA2R and b THSD7A were both positive along the glomerular capillaries
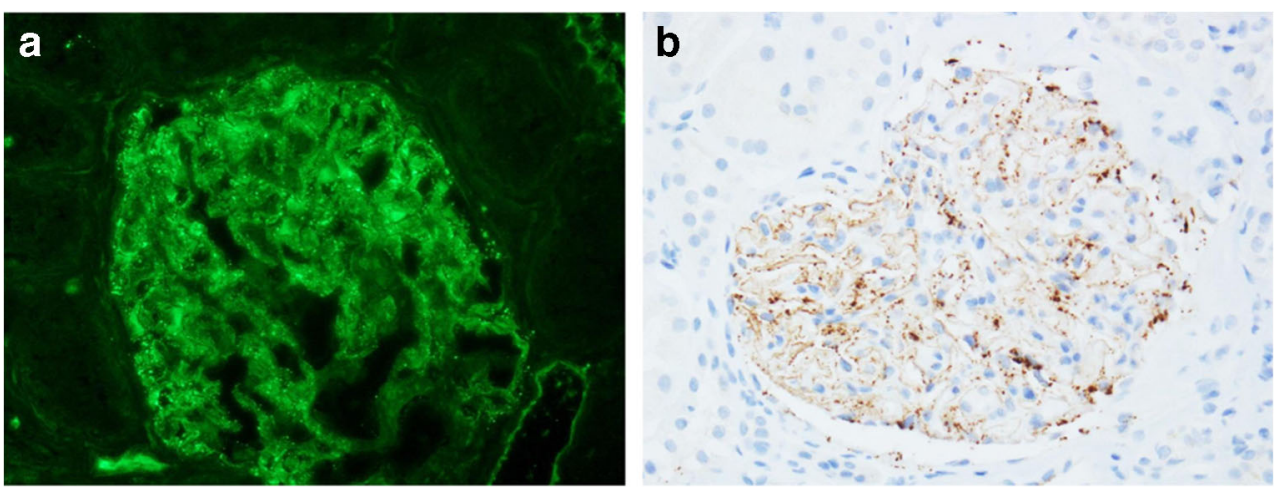


\section{IgG1}
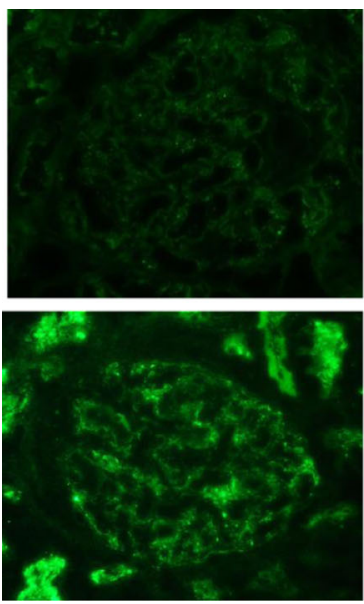

$\operatorname{IgG} 2$
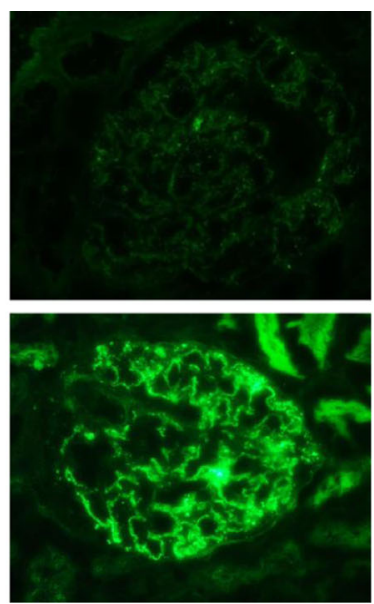

$\operatorname{IgG} 3$
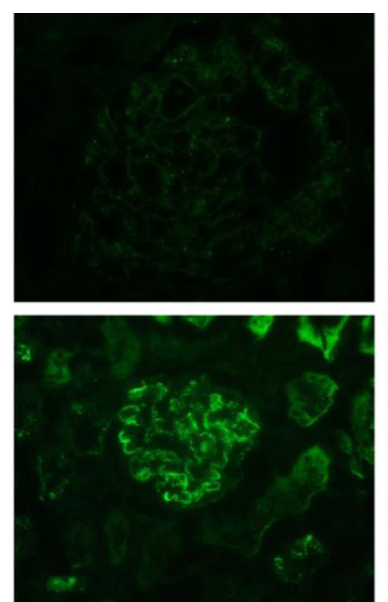

IgG4
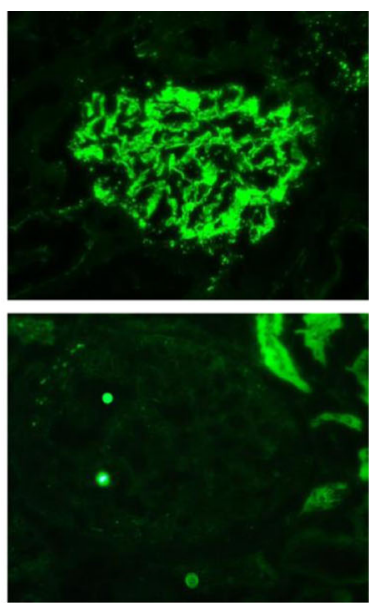

Fig. 3 Representative images of sections immunostained for IgG subclass profiling. IgG4-dominant (case 1) and IgG2-dominant distribution (case 14)

Similarly, the pathogenic association of THSD7A-associated MN with asthma (cases 10 to 12 ) remains unclear, although hypereosinophilic disorders other than Kimura's disease have been reported in association with MN [30, 31].

Alternatively, an association between malignancy and $\mathrm{MN}$ is well documented [32], and several pathological and laboratory findings have been proposed as predictive of malignancy, such as an increased number of intraglomerular inflammatory cells [33], an IgG subclass profile with IgG1 and IgG2 [34] and THSD7A-targetted serum autoantibodies or glomerular THSD7A positivity on immunostaining [14, 16]. Furthermore, a previous study on THSD7A-associated MN reported significantly increased numbers of glomeruli with $>8$ inflammatory cells [16]. In the present study, however, intraglomerular inflammatory cells were not evident in either case with malignancy. The case with lung cancer (case 13) demonstrated an IgG4-dominant profile of glomerular immune deposits, implying that cancerassociated $\mathrm{MN}$ is unlikely according to a previous report [34]. In the case with prostatic cancer (case 14), however, IgG2 was dominant and IgG4 was negative, suggesting a specific association with $\mathrm{MN}$ based on previous findings. Different profiles of lectin complement pathway components between THSD7A-associated MN cases with and without comorbid malignancy also suggest distinct pathogenic mechanisms (Supplementary Fig. 3). However, cancer tissues were THSD7A-negative by immunostaining, providing no evidence for a mechanistic association with $\mathrm{MN}$. The reason for this lack of association between cancer and THSD7A-associated MN in our study cohort remains elusive and requires further investigation. Nonetheless, given the relatively high rate of comorbidity ( 2 of 14 case), intensive cancer surveillance is warranted for THSD7Aassociated MN.
Table 3 Clinical follow-up and treatment

\begin{tabular}{lllll}
\hline Case & $\begin{array}{l}\text { Crat last follow-up } \\
(\mathrm{mg} / \mathrm{dl})\end{array}$ & $\begin{array}{l}\text { Proteinuria at last } \\
\text { follow-up }(\mathrm{g} / \mathrm{gCr})\end{array}$ & Treatment & $\begin{array}{l}\text { Observation } \\
\text { period (months) }\end{array}$ \\
\hline 1 & 1.28 & 4.29 & PSL, ARB, CNI, ATV & 34.0 \\
2 & 0.76 & 0.16 & PSL, ARB & 32.1 \\
3 & 0.94 & 0.07 & PSL & 58.4 \\
5 & 0.97 & 0.07 & NA & NA \\
8 & 1.03 & 1.40 & PSL, ARB & 3.1 \\
9 & 0.79 & 0.37 & PSL, ARB, ATV & 37.0 \\
10 & 0.63 & 0.11 & PSL, ARB & 62.6 \\
11 & 0.71 & 0.03 & NA & NA \\
13 & 0.92 & 0.03 & PSL & 49.9 \\
14 & 0.56 & 11.86 & no treatment & 43.0 \\
Average & 0.86 & 1.84 & - & 40.0 \\
\hline
\end{tabular}

$A R B$ angiotensin II receptor blocker, $A T V$ atorvastatin, $C N I$ calcineurin inhibitor, $C r$ creatinine, $N A$ not available, $P S L$ prednisone 
Light microscopic findings of the present study are in contrast with those of our previous study on PLA2R-associated MN, in which approximately $70 \%$ of cases demonstrated spike formation on light microscopic examination [7]. However, it is premature to draw the conclusion that inconspicuous manifestations of glomerular basement membranes are the common hallmark of THSD7A-associated MN. Indeed, Sharma et al. [35] examined the Ehrenreich and Churg classification of THSD7A-associated $\mathrm{MN}(n=26)$ : stage 1, 10 cases; stage 2, 8 cases; stage 3, 7 cases; stage 4,1 case. The morphological changes in the glomerular basement membrane in MN become apparent with time [36]. In our cohort, the average time elapsed between disease onset and pathological diagnosis was 3.4 (range, 2 to 8 ) months. Therefore, inconspicuous spike formation in most of our cases may be partly related to the early diagnosis of MN. However, we have no explanation for the lack of advanced cases of THSD7Aassociated $\mathrm{MN}$ in this study.

A heatmap plot of immunoglobulin and complement immunofluorescence demonstrated that $\mathrm{IgG}$ and $\mathrm{C} 3$ were the major immune complex components in THSD7A-associated $\mathrm{MN}$. In general, the IgG profile did not distinguish THSD7Aassociated from PLA2R-associated MN. In the present cohort, IgG4 dominant/codominant was the most common IgG subclass profile, similar to PLA2R-associated MN [7] and iMN in general. Therefore, the THSD7A-associated MN in this study also lacked glomerular features suggestive of sMN (Table 2). One case in the present study (case 2) showed dual positivity (PLA2R and THSD7A), which was also reported in previous studies from China [15] and the USA [17]. Although the pathogenesis of dual positivity remains unclear, the present study indicates that immunological reactions involving both PLA2R and THSD7A antigens can occur in different ethnic groups.

Activation of lectin complement pathway is associated with various renal diseases, including IgA vasculitis [37], poststreptococcal acute glomerulonephritis [38] and lupus nephritis [39]; however, association between THSD7A-related MN and lectin complement pathway has not been examined. The present study provides the first evidence that some cases of THSD7A-associated MN displays less significant activation of lectin complement pathway; we have no mechanistic insight regarding this possible association. Further, future studies using larger numbers of THSD7A-associated MN are required to validate the pathogenic mechanism.

In the present study cohort, prednisone therapy, alone or in combination with an angiotensin II receptor blocker, was effective for decreasing proteinuria. However, one case demonstrated a poor response despite combined therapy with prednisone, an angiotensin II receptor blocker, a calcineurin inhibitor and atorvastatin (case 1). Future prospective studies are required to elucidate the factors affecting the treatment responses of THSD7A-associated MN.

Limitations of this study include the small sample of THSD7A-associated MN cases. Small case numbers are an inherent limitation given the rarity of THSD7A-associated MN. Nationwide or transnational databases are required to fully characterise disease features, especially cases associated with malignancy. Second, we did not examine serum anti-THSD7A antibodies. Although THSD7A tissue staining of renal biopsy specimens strongly correlates with serum antibody testing [35], combined assessment of both serum anti-THSD7A antibodies and tissue THSD7A staining may further provide insights into the clinicopathological features of THSD7A-associated MN. Third, the retrospective design precludes establishing causal relationships between treatment and patient outcome.

In summary, our findings indicate that THSD7A-associated $\mathrm{MN}$ is characterised by (1) regional variation in prevalence (e.g. a higher prevalence in northern than southwestern Japan), (2) frequent nephrotic-range proteinuria, (3) a possible association with allergic disorders, (4) mainly IgG4 dominant/codominant IgG subclass profile and (5) a distinct profile of lectin complement pathway in rare subgroup. The associations between THSD7A-associated $\mathrm{MN}$ and malignancy require further investigation based on a nationwide disease registry.

Authors' contributions SH conceived the research idea and study design and performed data acquisition as well as data analysis/interpretation; TT and TH performed data acquisition as well as data analysis/interpretation; YF, SH, SM, SG and YA performed data acquisition and SN and TI supervised the work and provided mentorship. SH takes responsibility that this study has been reported honestly, accurately and transparently and accepts the accountability for the overall work by ensuring that questions pertaining to the accuracy or integrity of any portion of the work are appropriately investigated and resolved.

Funding This work was supported by MEXT KAKENHI (Grant Number JP 17 K08741).

\section{Compliance with ethical standards}

The institutional review board of Kobe City Medical Center General Hospital approved the study protocol (No. 180635).

Conflict of interest The authors declare that they have no conflict of interest.

Open Access This article is distributed under the terms of the Creative Commons Attribution 4.0 International License (http:// creativecommons.org/licenses/by/4.0/), which permits unrestricted use, distribution, and reproduction in any medium, provided you give appropriate credit to the original author(s) and the source, provide a link to the Creative Commons license, and indicate if changes were made.

\section{References}

1. Beck LH Jr, Salant DJ (2010) Membranous nephropathy: recent travels and new roads ahead. Kidney Int 77:765-770

2. Beck LH Jr, Bonegio RG, Lambeau G, Beck DM, Powell DW, Cummins TD, Klein JB, Salant DJ (2009) M-type phospholipase 
A2 receptor as target antigen in idiopathic membranous nephropathy. N Engl J Med 361:11-21

3. Qin W, Beck LH Jr, Zeng C, Chen Z, Li S, Zuo K, Salant DJ, Liu Z (2011) Anti-phospholipase A2 receptor antibody in membranous nephropathy. J Am Soc Nephrol 22:1137-1143

4. Hoxha E, Harendza S, Zahner G, Panzer U, Steinmetz O, Fechner K, Helmchen U, Stahl R (2011) An immunofluorescence test for phopholiase-A2-receptor antibodies and its clinical usefulness in patients in membranous glomerulonephritis. Nephrol Dial Transplant 26:2526-2532

5. Akiyama S, Akiyama M, Imai E, Ozaki T, Matsuo S, Maruyama S (2015) Prevalence of anti-phospholipase A2 receptor antibodies in Japanese membranous nephropathy. Clin Exp Nephrol 19:653-660

6. Hayashi N, Akiyama S, Okuyama H, Matsui Y, Adachi Y, Yamaya H, Maruyama S, Imai E, Matsuo S, Yokoyama H (2015) Clinicopathological characteristics of M-type phospholipase A2 receptor (PLA2R)-related membranous nephropathy in Japanese. Clin Exp Nephrol 19:797-803

7. Hara S, Goto S, Kamiura N, Yoshimoto A, Naito T, Imagawa N, Imai Y, Yanagita M, Nishi S, Ito T (2015) Reappraisal of PLA2R1 in membranous nephropathy: immunostaining method influence and association with IgG4-dominant phenotype. Virchows Arch 467:87-94

8. Bech AP, Hofstra JM, Brenchley PE, Wetzels JF (2015) Association of anti-PLA(2)R antibodies with outcomes after immunosuppressive therapy in idiopathic membranous nephropathy. Clin J Am Soc Nephrol 9:1386-1392

9. Kanigicherla D, Gummadova J, McKenzie EA, Roberts SA, Harris S, Nikam M, Poulton K, McWilliam L, Short CD, Venning M, Brenchley PE (2013) Anti-PLA2R antibodies measured by ELISA predict long-term outcome in a prevalent population of patients with idiopathic membranous nephropathy. Kidney Int 83: 940-948

10. Hoxha E, Thiele I, Zahner G, Panzer U, Harendza S, Stahl RA (2014) Phospholipase A2 receptor autoantibodies and clinical outcome in patients with primary membranous nephropathy. J Am Soc Nephrol 25:1357-1366

11. Quintana LF, Blasco M, Seras M, Pérez NS, López-Hoyos M, Villarroel P, Rodrigo E, Viñas O, Ercilla G, Diekmann F, GómezRoman JJ, Fernandez-Fresnedo G, Oppenheimer F, Arias M, Campistol JM (2015) Antiphospholipase A2 receptor antibody levels predict the risk of posttransplantation recurrence of membranous nephropathy. Transplantation 99:1709-1714

12. Tomas NM, Beck LH Jr, Meyer-Schwesinger C, Seitz-Polski B, Ma H, Zahner G, Dolla G, Hoxha E, Helmchen U, Dabert-Gay AS, Debayle D, Merchant M, Klein J, Salant DJ, Stahl RAK, Lambeau G (2014) Thrombospondin type-1 domain-containing 7A in idiopathic membranous nephropathy. N Engl J Med 371: 2277-2287

13. Iwakura T, Ohasni N, Kato A, Baba S, Yasuda H (2015) Prevalence of enhanced granular expression of thrombospondin type-1 domain-containing 7A in the glomeruli of Japanese patients with idiopathic membranous nephropathy. PLoS One 10: e0138841

14. Hoxha E, Beck LH Jr, Wiech T, Tomas NM, Probst C, Mindorf S, Meyer-Schwesinger C, Zahner G, Stahl PR, Schöpper R, Panzer U, Harendza S, Helmchen U, Salant DJ, Stahl RA (2017) An indirect immunofluorescence method facilitates detection of thrombospondin type 1 domain-containing 7A-specific antibodies in membranous nephropathy. J Am Soc Nephrol 28:520-531

15. Wang J, Cui Z, Lu J, Probst C, Zhang YM, Wang X, Qu Z, Wang F, Meng LQ, Cheng XY, Liu G, Debiec H, Ronco P, Zhao MH (2017) Circulating antibodies against thrombospondin type-I domain-containing 7A in Chinese patients with idiopathic membranous nephropathy. Clin J Am Soc Nephrol 12:1642-1651
16. Hoxha E, Wiech T, Stahl PR, Zahner G, Tomas NM, MeyerSchwesinger C, Wenzel U, Janneck M, Steinmetz OM, Panzer U, Harendza S, Stahl RA (2016) A mechanism for cancer-associated membranous nephropathy. N Engl J Med 374:1995-1996

17. Larsen CP, Cossey LN, Beck LH (2016) THSD7A staining of membranous glomerulopathy in clinical practice reveals cases with dual autoantibody positivity. Mod Pathol 29:421-426

18. Larsen CP, Messias NC, Silva FG, Messias E, Walker PD (2013) Determination of primary versus secondary membranous glomerulopathy utilizing phospholipase A2 receptor staining in renal biopsies. Mod Pathol 26:709-715

19. Ehrenreich T, Churg J (1968) Pathology of membranous nephropathy. In: Sommers SC, ed. Pathology Annual vol 3. New York: Appleton-Century-Crofts, 1968:145-154

20. Kaga H, Komatsuda A, Omokawa A, Okuyama S, Mori K, Wakui H, Takahashi N (2018) Analysis of PLA2R1 and HLA-DQA1 sequence variants in Japanese patients with idiopathic and secondary membranous nephropathy. Clin Exp Nephrol 22:275-282

21. Thiri M, Honda K, Kashiwase K, Mabuchi A, Suzuki H, Watanabe K, Nakayama M, Watanabe T, Doi K, Tokunaga K, Noiri E (2016) High-density association mapping and interaction analysis of PLA2R1 and HLA regions with idiopathic membranous nephropathy in Japanese. Sci Rep 6:38189

22. Bomback AS (2018) Management of membranous nephropathy in the PLA ${ }_{2} \mathrm{R}$ era. Clin J Am Soc Nephrol 13:784-786

23. Sethi S, Haas M, Markowitz GS, D'Agati VD, Rennke HG, Jennette JC, Bajema IM, Alpers CE, Chang A, Cornell LD, Cosio FG, Fogo AB, Glassock RJ, Hariharan S, Kambham N, Lager DJ, Leung N, Mengel M, Nath KA, Roberts IS, Rovin BH, Seshan SV, Smith RJ, Walker PD, Winearls CG, Appel GB, Alexander MP, Cattran DC, Casado CA, Cook HT, De Vriese AS, Radhakrishnan J, Racusen LC, Ronco P, Fervenza FC (2016) Mayo Clinic/Renal Pathology Society consensus report on pathologic classification, diagnosis, and reporting of GN. J Am Soc Nephrol 27:1278-1287

24. Yamada A, Mitsuhashi K, Miyakawa Y, Kosaka K, Takehara K, Iijima M, Tanaka K, Shibata S (1982) Membranous glomerulonephritis associated with eosinophilic lymphfolliculosis of the skin (Kimura's disease): report of a case and review of the literature. Clin Nephrol 18:211-215

25. Akosa AB, Sherif A, Maidment CG (1991) Kimura's disease and membranous nephropathy. Nephron 1991;58:472-474

26. Obata Y, Furusu A, Nishino T, Ichinose H, Ohnita A, Iwasaki K, Taguchi T, Kohno S (2010) Membranous nephropathy and Kimura's disease manifesting a hip mass. A case report with literature review. Intern Med 49:1405-1409

27. Chen Y, Wang J, Xu F, Zeng C, Liu Z (2016) Clinicopathological features and prognosis of Kimura's disease with renal involvement in Chinese patients. Clin Nephrol 85:332-339

28. Gaillard J, Rotman S, Girardet C, Spertini F (2017) Successful therapy with mycophenolic acid in a membranous glomerulonephritis due to Kimura disease. Clin Nephrol 88:221-225

29. Okura T, Miyoshi K, Irita J, Enomoto D, Nagao T, Kukida M, Tanino A, Kudo K, Higaki J (2014) Kimura's disease associated with membranous nephropathy with IgG4 and phospholipase A2 receptor-positive staining of the glomerular basement membrane. Intern Med 53:1435-1440

30. Frigui M, Hmida MB, Jallouli M, Kechaou M, Frikha F, Bahloul Z (2010) Membranous glomerulopathy associated with idiopathic hypereosinophilic syndrome. Saudi J Kidney Dis Transpl 21:320 322

31. Lanfranchi J, Meyrier A, Sachs RN, Guillevin L (1986) Blood hypereosinophilia syndrome with cardiac involvement and extramembranous glomerulopathy. Ann Med Interne (Paris) 137: $133-137$ 
32. Leeaphorn N, Kue-A-Pai P, Thamcharoen N, Ungprasert P, Stokes MB, Knight EL (2014) Prevalence of cancer in membranous nephropathy: a systematic review and meta-analysis of observational studies. Am J Nephrol 40:29-35

33. Lefaucheur C, Stengel B, Nochy D, Martel P, Hill GS, Jacquot C, Rossert J, GN-PROGRESS Study Group (2006) Membranous nephropathy and cancer: epidemiologic evidence and determinants of high-risk cancer association. Kidney Int 70:1510-1517

34. Ohtani H, Wakui H, Komatsuda A, Okuyama S, Masai R, Maki N, Kigawa A, Sawada K, Imai H (2004) Distribution of glomerular IgG subclass deposits in malignancy-associated membranous nephropathy. Nephrol Dial Transplant 19:574579

35. Sharma SG, Larsen CP (2017) Tissue staining for THSD7A in glomeruli correlates with serum antibodies in primary membranous nephropathy: a clinicopathological study. Mod Pathol 31:616-622
36. Couser WG (2018) Primary membranous nephropathy. Clin J Am Soc Nephrol 7:983-997

37. Hisano S, Matsushita M, Fujita T, Iwasaki H (2005) Activation of the lectin complement pathway in Henoch-Shonlein purpura nephritis. Am J Kidney Dis 45:295-302

38. Hisano S, Matsushita M, Fujita T, Takeshita M, Iwasaki H (2007) Activation of the lectin complement pathway in post-streptococcal acute glomerulonephritis. Pathol Int 57:351-357

39. Nishihara RM, Magrini F, Mocelin V, Messias-Reason IJ (2013) Deposition of the lectin pathway of complement in renal biopsies of lupus nephritis patients. Hum Immunol 74:907-910

Publisher's note Springer Nature remains neutral with regard to jurisdictional claims inpublished maps and institutional affiliations. 